\title{
Non-malignant Oral Ulcer: A Diagnostic Challenge to Otorhinolaryngologists
}

\author{
Neeraj Aggarwal, ${ }^{1}$ Sirshak Dutta, ${ }^{2}$ Avijit Choudhury, ${ }^{3}$ Mainak Dutta, ${ }^{4}$ Saumik Das, ${ }^{4}$ Ramanuj Sinha ${ }^{4}$
}

\section{Introduction}

\section{$\underline{\text { ABSTRACT }}$}

Apart from the malignant ulcers a wide variety of dermatological and other systemic conditions can affect oral cavity and often be a diagnostic challenge due to their similar presenting features and appearance. Despite being benign, some of them may be fatal if not diagnosed and treated timely. This study aims to assess the incidence of different non malignant ulcers in oral cavity and to estimate the need for histopathological examination for confirmation of the condition for proper management. Materials and Methods

An institution based observational study was conducted over a time period of 16 months at a tertiary care institute. Total 172 cases were included in the present study irrespective of age, sex and duration. Malignant lesions were excluded from the study. $\underline{\text { Results }}$

The present study shows a slight male preponderance $(M: F=1.48: 1)$ with majority of cases were in age group 20-40 years of age $(43.02 \%)$. Most commonly encountered were recurrent aphthous stomatitis (27.9\%) followed by oral lichen planus (16.28\%), oral candidiasis, angular stomatitis, traumatic ulcers and others. $78.5 \%$ were treated successfully on the basis of clinical diagnosis while $21.5 \%$ cases needed further histopathological or other investigation for confirmation of diagnosis.

\section{Conclusion}

Though the otolaryngologists are more concerned about malignant ulcer, there are many non malignant ulcers which can cause significant morbidity and can even be fatal and should be given similar importance like malignant lesions. Majority of them can be treated successfully on the basis of clinical diagnosis but histopathological examination may be required in some cases for proper diagnosis and appropriate management.

Keywords

Oral Ulcer; Benign

$\mathrm{O}$ ral ulcer is not a very uncommon entity for an Otorhinolaryngologist. A significant number of patients with oral ulcers attend Otolaryngology outpatient department. Non-malignant ulcer is an underdiscussed spectrum of disease, but can cause significant morbidity and sometimes can even be fatal. The major contribution among the oral ulcers is from the nonmalignant lesions which also need to be diagnosed timely. Maximum emphasis was put on malignant ulcers in the past as evident from available literature. Oral ulcers may seem to be a simple problem; however, it raises many questions about the differential diagnoses of neoplastic, inflammatory, traumatic, and autoimmune conditions involving the oral cavity.

Different types of oral ulcers presented with similar symptoms leading to diagnostic difficulties. Because of the diagnostic dilemma in identifying the cause of the persistently annoying symptoms suffered by the patient of non-malignant oral ulcers and failure of repeated biopsies to diagnose the situation, the treatment for the same is delayed. With this background, an observational study was conducted in a tertiary care centre, to assess the incidence, demographic characteristics, different presentations and diagnosis, clinical and histopathological correlation of the patient presenting

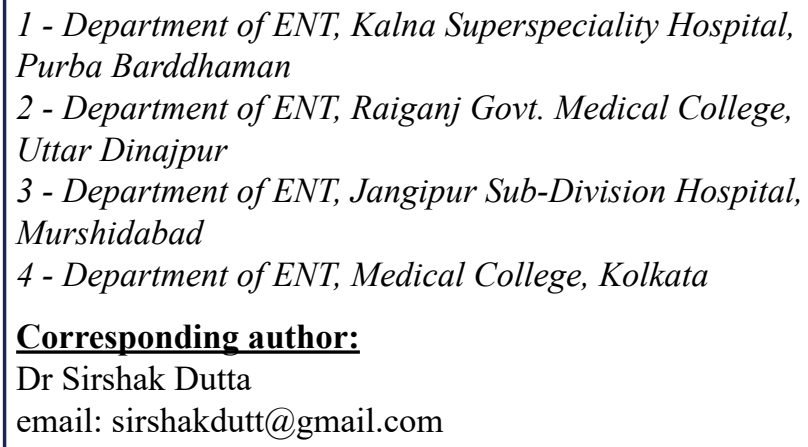




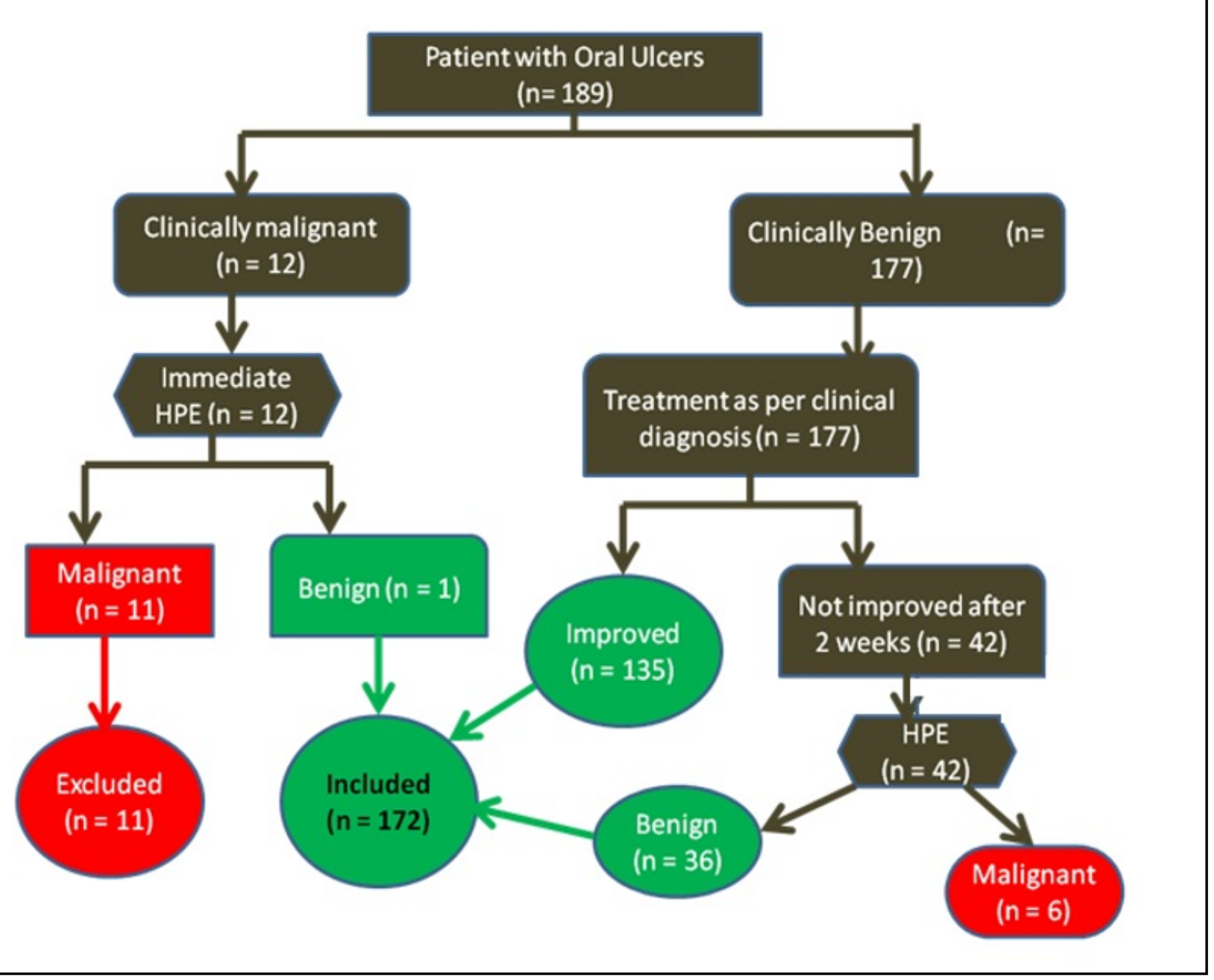

Fig. 1. Working algorithm for inclusion of cases

with non-malignant oral ulcer.

\section{Materials and Methods}

A descriptive observational study was conducted in a tertiary care hospital, on patients attending Otolaryngology outpatient department (OPD) with oral ulcers in a period between November 2015-February 2017. These patients underwent proper clinical evaluation. Out of 189 cases of oral ulcers, 172 cases were clinically or histologically confirmed to be benign and are included in the study. Those patients who were suspected to be non-malignant clinically were given an empirical treatment based on the clinical diagnosis for two weeks. If the lesion still persisted, incision biopsy from the ulcers were sent for histopathological evaluation. In some cases, special investigations in the form of Anti-nuclear antibodies (ANA), Anti-Ds DNA, Direct immune florescence, sputum examination, Chest X-ray, etc. were done. On the other hand, all the clinically suspected malignant ulcers underwent histopathological evaluation immediately. One case in this group was found to be benign and was included in the study. (Fig. 1) 


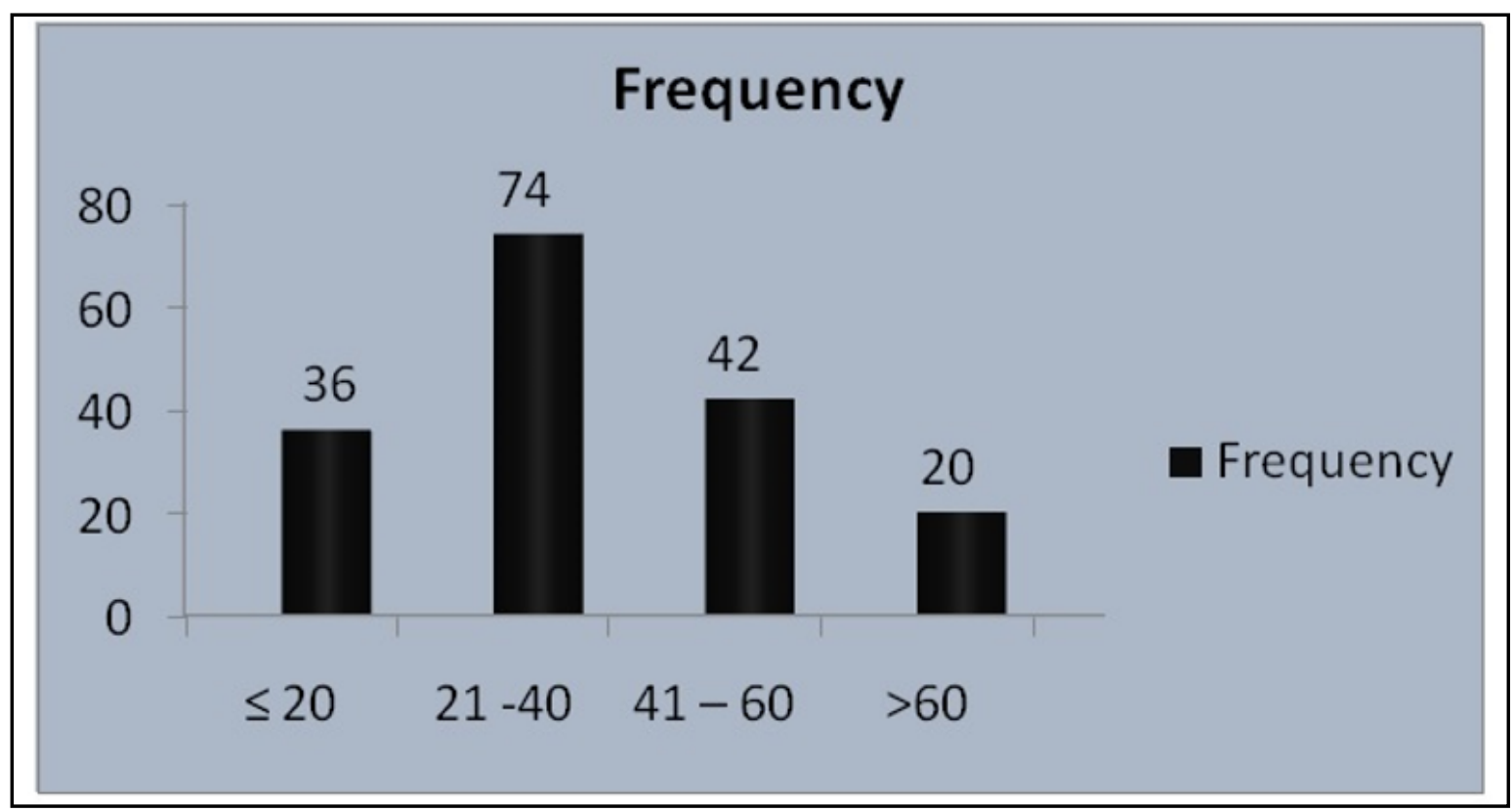

Fig. 2. Age distribution of the patients

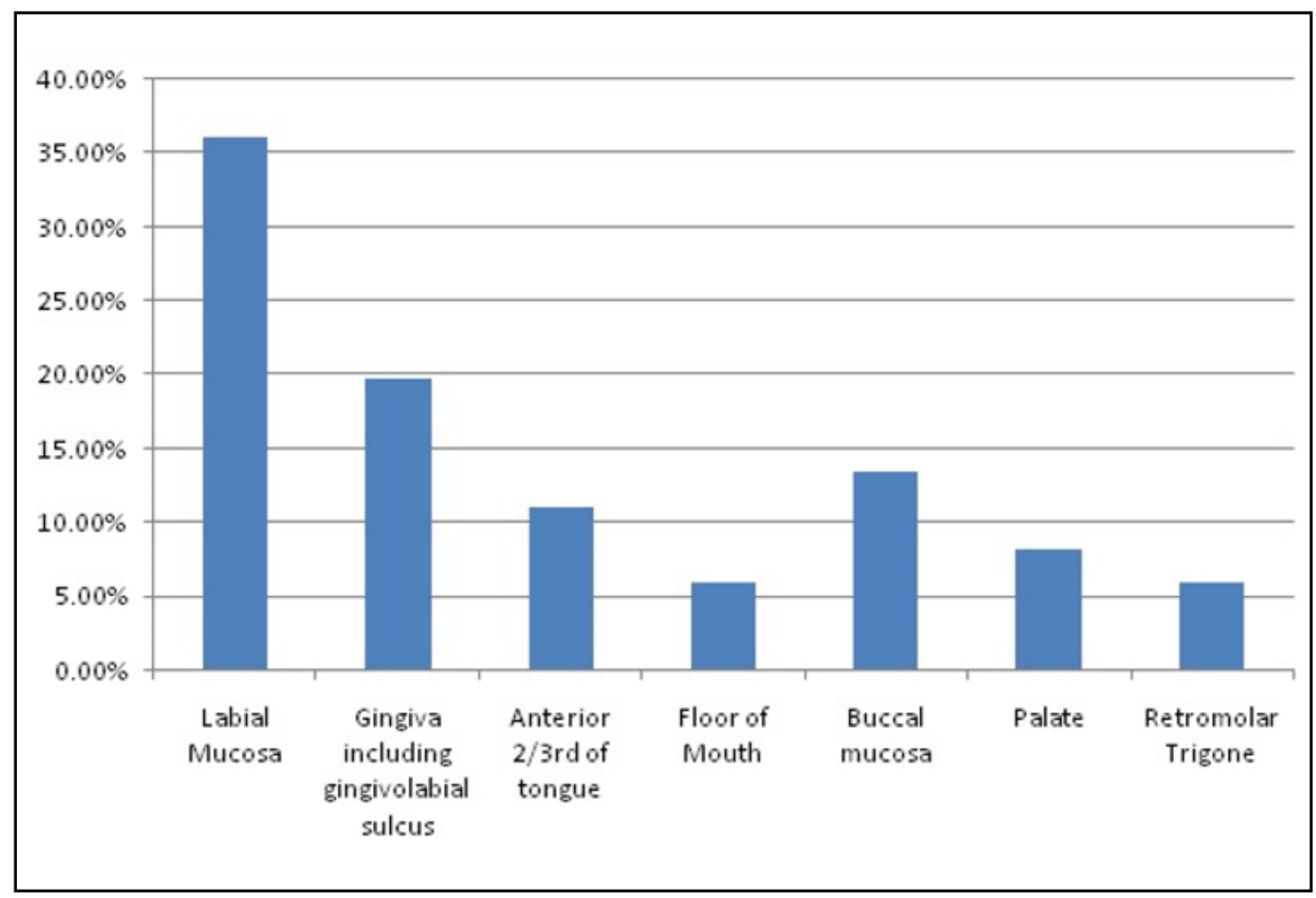

Fig. 3. distribution of case according to site of occurrence 


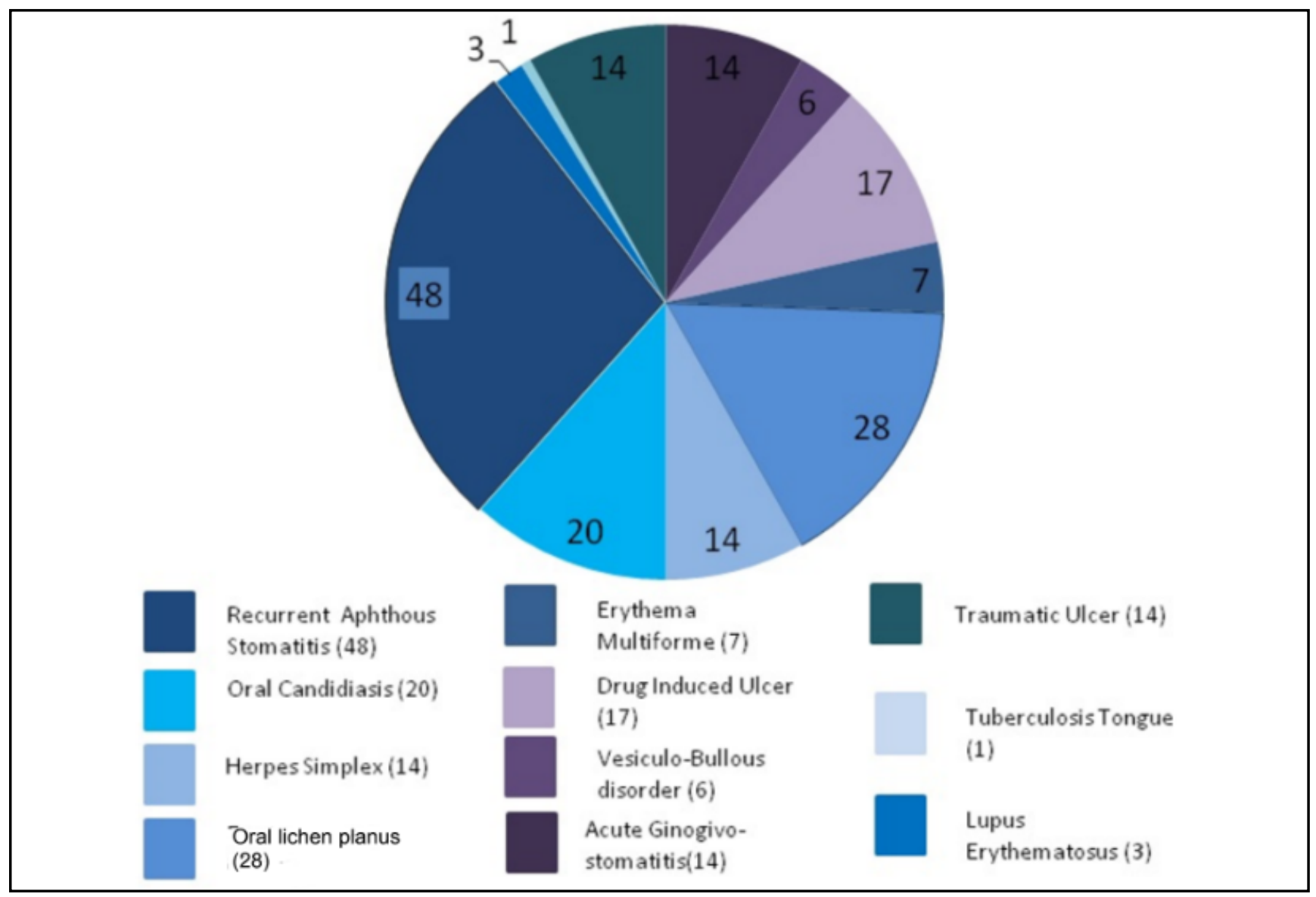

Fig. 4. Variety of ulcers detected in oral cavity

\section{Results}

Among 189 cases of oral ulcer, 172 (91\%) were diagnosed to be non-malignant. (Fig. 1)

In the present study most of the patients of nonmalignant ulcer were in the age group of 20-40 years $(43.02 \%)$ followed by the age group $40-60$ years. (Fig. 2)

There was a slight male preponderance (102 cases, i.e. 59.3\%) among the cases of non malignant oral ulcer.

Though there was significant history of addiction in the study population ( 142 cases i.e. $82.56 \%$ ) to one or more substance of abuse, the association substance abuse to particular cause of non-malignant ulcer is beyond the limit of the study.

Among the 172 cases finally included in the study, 135 cases $(78.5 \%)$ were treated successfully on the basis of the clinical diagnosis, while 37 cases $(21.5 \%)$ required histopathological examination for confirmation or special investigations for diagnosis. (Fig. 1)

While considering the site related preponderance of non malignant oral ulcer, labial mucosa was involved in almost $1 / 3$ rd of the patients (36\%) followed by gingiva including gingivolabial and gingivobuccal sulcus. (Fig. 3)

Most common lesion was recurrent aphthous stomatitis (27.9\%) followed by oral lichen planus. Many rare entities have been encountered during the study like tuberculosis of tongue, pemphigus vulgaris and many others. (Figs 4 \& 5)

\section{Discussion}

Most of the studies on oral health give more stress on 


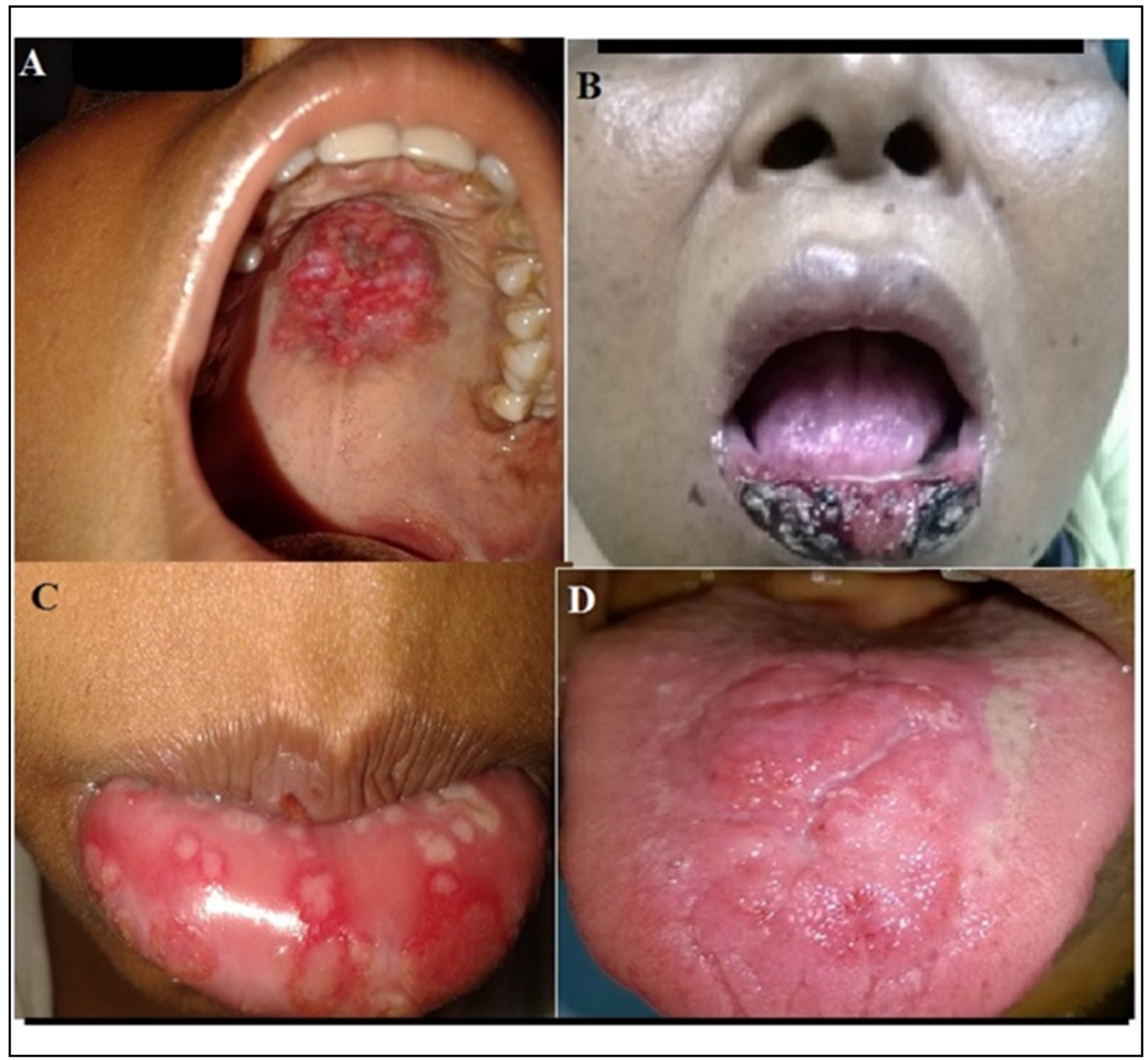

Fig. 5. A) Lupus Erythematosus, B) Pemphigus Vulgaris, C) Multiple Aphthous Stomatitis, D) Tuberculosis Tongue

dental issues and malignant disease of oral cavity and there is paucity of studies on non-malignant ulcer. ${ }^{1}$ Ahmed and Uddin (2010), in 'Oral ulceration at primary care' estimated the point prevalence of oral ulcer to be $4 \%$ worldwide. ${ }^{2}$ Though most of the treating physicians are concerned about the probability of malignancy in a patient of oral ulcer, the present study showed that most of the oral ulcers are benign (91\%). The age distribution of patients of oral ulcers varies from study to study and lesion to lesion. According to Roy and Varshney (2013) oral mucosal problems are most prevalent in the age group of 11-40 years. ${ }^{3}$ In the present study, most cases were in age group of 20-40 years (43.02\%).

If the gender distribution is considered, Saraswathi 
et al in their study concluded a slight preponderance of male with male: female ratio of $1.75: 1,{ }^{4}$ whereas Roy and Varshney found female preponderance for the condition with sex ratio of 2:3.3 In our study we encountered more male patients with a male: female ratio of 1.46:1. Roy and Varshney (2013), found in their study conducted on 150 cases, aphthous ulcer $(28.57 \%)$ and pemphigus vulgaris $(26.60 \%)$ formed the bulk of patients, followed by systemic lupus erythematosus $(17.02 \%)$, oral candidiasis $(16.07 \%)$, discoid lupus erythematosus (13.83\%), lichen planus $(12.77 \%)$ and others. $^{3}$

Different studies on oral mucosal lesions show varying results about the final diagnosis. Simi et al in their study on oral mucosal lesions concluded that oral Lichen planus (64\%) was the most common entity, ${ }^{5}$ while Bhatnagar et al in their study found prevalence of oral lichen planus to be $0.8 \%$, much less than recurrent aphthous stomatitis $(1.53 \%)$ and oral candidiasis $(1.61 \%){ }^{6}$ This shows the diversity of diagnosis of oral ulcer in different study groups. In present study, recurrent aphthous stomatitis $(27.9 \%)$ was most prevalent followed by erosive lichen planus $(16.28 \%)$.

Most of the cases of non-malignant oral ulcer were treated successfully on the basis of clinical diagnosis alone and less than one fourth cases needed histopathological or other confirmatory investigations. But it is evident from the study that histopathological examination in time according to the clinical nature and course of the disease is mandatory. This is not only to diagnose the malignant lesions, but also for precise diagnosis of some benign entities which need specific treatment on the basis of histopathology.

\section{Conclusion}

Every case of non-malignant oral ulcer is needed to be thoroughly examined and even rarest of the rare entities should be kept in mind while dealing with a case of non-malignant oral ulcer. It is to be kept in mind that not only the malignant ulcer but also many non-malignant oral ulcers, if not diagnosed timely, can be life threatening. To diagnose these life-threatening conditions like tuberculosis, pemphigus vulgaris or lupus erythromatosus, a high degree of clinical suspicion along with some timely special investigations should be done. In case of any diagnostic dilemma a team approach should be applied, including Dermatologist, Oral Pathologist and Otolaryngologist as oral ulcerations may be the manifestation of some underlying systemic diseases.

\section{References}

1. Petersen PE, Bourgeois D, Ogawa H, Day SE, Ndiaye C. The global burden of oral diseases and risks to oral health. Bulletin of the World Health Organization 2005; 83:661-9

2. Ahmed MU, Uddin MN. Oral ulceration at primary care -A Review. Bangladesh Journal of Plastic Surgery. 2010 ;1:23-9

3. Roy S, Varshney S. Oral dermatological conditions: A Clinical Study. Indian J Otolaryngol Head Neck Surg. 2013; 65:97-101

4. Saraswathi TR, Ranganathan K, Shanmugam S, Ramesh S, Narasimhan PD, Gunaseelan R. Prevelance of oral lesions in relation to habits: Cross-sectional study in south India. Ind J Dent Res. 2006; 17:121-5

5. Simi SM, Nandakumar G, Anish TS. White Lesions in the Oral Cavity: A clinicopathological study from a tertiary care dermatology centre in Kerala. Indian J Dermatol. 2013; 58:269-74

6. Bhatnagar P, Rai S S, Bhatnagar G, Kaur M, Goel S, Prabhat M. Prevalance study of oral mucosal lesions, mucosal variants, and treatment required for patients reporting to a dental school in north India: in accordance with WHO guide lines. J Family Community Med. 2013; 20: 41-8. 